\title{
Determination of Nicotine Exposure Using Passive Sampler and High Performance Liquid Chromatography
}

\author{
1 University of Shizuoka, Shizuoka, Japan \\ ${ }^{2}$ Seikei University, Musashino city, Tokyo, Japan \\ ${ }^{3}$ Yokohama National University, Yokohama, Japan
}

Amagai $\mathrm{T}^{1^{*}}$, Bai $\mathrm{H}^{1}$, Wang $\mathbf{Q}^{1}$, Miyake $\mathbf{Y}^{1}$, Noguchi $\mathbf{M}^{2}$ and Nakai $\mathbf{S}^{3}$

\begin{abstract}
To measure nicotine personal exposure in our daily life, we have developed a determination method of personal exposure to nicotine. The method consisted of passive sampling of nicotine, simple solvent extraction and high performance liquid chromatography (HPLC) determination. The passive sampler was badge type and collection media was sodium bisulfate impregnated quartz fiber filter. The collected nicotine as nicotine bisulfate was extracted with pure water simply. Nicotine bisulfate was separated by HPLC, and detected with spectrophotometry. Three kinds of HPLC separation column were tested, and the best separation was attained with a cation exchange column, Zorbax 300-SCX. We have also evaluated the kinds of filter used, stability of nicotine bisulfate. Nicotine bisulfate on the filter was not evaporated or degraded in the outdoor air. The amount of collected nicotine was converted to air concentration by the equation that was made from simultaneous active- and passive- sampling experiments. Then it was suggested that nicotine determination method developed in this study was useful for estimate nicotine personal exposure accurately.
\end{abstract}

Keywords: Nicotine; Personal exposure; Passive sampling; HPLC

\section{Introduction}

Passive smoking is one of the major public health problems in the world. Lung cancer is the cancer of the first place of cause-ofdeath ranking in Japan [1]. International agency for research on cancer classified not only tobacco smoking but also involuntary smoking into human carcinogen (Table 1) [2]. By Health Promotion Act in Japan, it was restricted to smoke tobacco at a public place in Japan. A similar law was made by developed countries. Exposure to ETS for non-smoker was important problems in the world [3].

To estimate health effect of ETS, it is necessary to measure personal exposure to environmental tobacco smoke (ETS) quantitatively. Nicotine is a well-known marker of ETS as well as particulate matter $[4,5]$. The standard method for determining vapor phase nicotine was published by international standard organization (ISO 18145) [6]. In this method, nicotine was collected actively by XAD- 4 beads packed in the tube, extracted with ethyl acetate and analyzed by GC/NPD. Since this method used sampling pump, it was difficult to be used for personal exposure measurement. It was also reported that nicotine was evaporated or degraded during sampling when the sampling time was longer. Saito et al. reported that nicotine collection amount decrease during sampling when adsorbent such as XAD-4 was used for collection, and they used alkaline coating XAD-4 to prevent decreasing of nicotine during sampling [7].

Passive sampler is small, light, noiseless and cheap, then it is suitable for analyze personal exposure for air pollutants [8]. It was reported

\begin{tabular}{|c|c|c|c|}
\hline I & Recovery & \pm & SD \\
\hline Quartz fiber filter (QR-100) & $94 \%$ & \pm & $11 \%$ \\
\hline Glass fiber filter (GA55) & $49 \%$ & \pm & $27 \%$ \\
\hline Glass fiber filter (GB100) & $25 \%$ & \pm & $15 \%$ \\
\hline
\end{tabular}

SD: Standard Deviation

Table 1: Recoveries of nicotine from different filters. that sodium bisulfate impregnated filter was useful for collecting nicotine [9]. Recently, MoNIC passive sampler was developed by Huynh et al., for determining personal exposure to nicotine [10,11]. In this method, nicotine was collected as sulfate in the sampler. Then the sample was neutralized by alkaline solution, and nicotine in the sample was extracted by organic solvent and determined by GC/MS .

Nicotine was determined with reverse phase HPLC in several papers [12-14]. Ion pair HPLC was also used [15]. Then we found that nicotine salts could be determined with HPLC/spectrometry.

We have developed an analytical method including extraction of nicotine sulfate by distilled water and analysis of the aqueous solutions by HPLC. Since we determined nicotine sulfate directly by HPLC / spectrophotometry in this system, the operations were simple.

\section{Materials and Methods}

\section{Reagents}

Reagent grade nicotine was purchased from Wako Pure Chemical Industries Ltd., Osaka. HPLC grade methanol, reagent grade sodium bisulfate, ammonium formate, quinoline and trimethylamine were also bought from Wako. Distilled water for $0.5 \%$ ammonium formate solution was purified with Milli Q Direct Q-UV (Merck KGaA, Darmstadt, Germany).

*Corresponding author: Amagai T, Department of Environmental Science and Health, University of Shizuoka, Shizuoka, Japan, Tel: + (81)-54-264-5798; E-mail: amagai@u-shizuoka-ken.ac.jp

Received July 16, 2015; Accepted July 29, 2015; Published July 31, 2015

Citation: Amagai T, Bai H, Wang Q, Miyake Y, Noguchi M, et al. (2015) Determination of Nicotine Exposure Using Passive Sampler and High Performance Liquid Chromatography. Pharm Anal Acta 6: 399. doi:10.4172/21532435.1000399

Copyright: @ 2015 Amagai T, et al. This is an open-access article distributed under the terms of the Creative Commons Attribution License, which permits unrestricted use, distribution, and reproduction in any medium, provided the original author and source are credited. 


\section{The passive sampler}

The passive sampler consisted of sodium bisulfate impregnated filter and filter holder. The size of the filter was 25 or $47 \mathrm{~mm} \Phi$. We have tested 3 types of collection filter; glass fiber filter GB-100R, GA-55, and quartz fiber filter QR-100 (Toyo Roshi Kaisha, Ltd., Tokyo). These collection filters was dipped in sodium bisulfate aqueous solution, dried and set in the filter holder. The filter holder was for asbestos (080140076, Shibata Sci. Technol., Tokyo). Each sampler was put into the aluminum/polyethylene bag and sealed.

\section{Extraction and HPLC determination}

Nicotine was collected as nicotine sulfate. After sampling, the filter was put into $10 \mathrm{~mL}$ of the test tube. Purified water treated with Direct-Q (Merck KGaA, Darmstadt, Germany) was added to it. The sample was ultrasonicated for 10 minutes, and centrifuged at $3000 \mathrm{rpm}$ for 10 minutes. The supernatant was taken to vial for autosampler of HPLC.

HPLC apparatus was Infinity 1260 (Agilent, Tokyo) with a diode array detector (DAD). The wavelength for quantification of nicotine bisulfate was $254 \mathrm{~nm}$.

Three kinds of HPLC column was tried; standard ODS column, Hilic column and ion exchange column. Standard ODS column was Poroshell $120 \mathrm{EC}-\mathrm{C} 182.7 \mu \mathrm{m}(4.6 \mathrm{~mm} \Phi, 50 \mathrm{~mm}$ in length, Agilent, Tokyo), Hilic column was Poroshell 120 HILIC (2.1 $\mathrm{mm} \Phi, 100 \mathrm{~mm}$ in length, Agilent, Tokyo), and ion exchange column was ZORBAX 300SCX (2.1 mm $\Phi, 150 \mathrm{~mm}$ in length, Agilent, Tokyo). Mobile phases of the HPLC were methanol and $0.5 \%$ ammonium formate: $80 \%$ methanol for $2 \mathrm{~min}$ at the beginning, and $80 \%$ methanol to $70 \%$ methanol in 2.6 minutes. There was no nicotine blank in the system.

\section{Quality assurance/quality control}

The method detection limit was defined as 3 times of the standard deviation of the nicotine amount when we analyzed the dilute standard solution repeatedly. Field blank samples were taken at least $10 \%$ of the sample number. Reproducibility of the nicotine analysis was performed using 6 samplers. These samplers were simultaneously exposed to the air in outdoor smoking area for one hour, and analyzed as above.

\section{Stability test of nicotine bisulfate during sampling}

To estimate stability of nicotine during sampling, the sampler was exposed to clean outdoor air after nicotine sampling. The outdoor concentration was below detection limit. After 8 samplers were simultaneously exposed to ETS polluted indoor air for one hour, 2 of them were extracted at once, and the rests were exposed to clean outdoor air. Two of them were extracted after 2, 7, and 24 hours.

\section{Conversion of nicotine collection amount to air concentration}

The amount of 1,3-butadiene collected with the passive sampler were converted to air concentration using a conversion equation. To make the conversion equation, active- and passive- sampling experiments were performed simultaneously in the residential room where one to for cigarettes was smoked. Active sampler was consisted of XAD-4 tube and minipump (Sibata Sci. and Technol. Inc., Tokyo). Sampling rate was $0.20 \mathrm{~L} / \mathrm{min}$, and sampling was performed for 1 hour. After sampling, nicotine in XAD- 4 was extracted with ethyl acetate containing $0.01 \%$ of triethylamine, and quinoline was used as an internal standard. Nicotine solution was determined by GC/MS. GC apparatus was Agilent 6890 GC with 5795 MSD (Agilent, Tokyo). GC column was DB-5 (30 m in length $0.25 \mathrm{~mm} \Phi, 0.25 \mu \mathrm{m}$ film thickness, Agilent, Tokyo). Column temperature was $50^{\circ} \mathrm{C}$ for 5 minutes, then rose at $10^{\circ} \mathrm{C} \cdot \mathrm{min}^{-1}$ to $220^{\circ} \mathrm{C}$. Detection mode was selected ion monitoring (SIM), and quantifying ion of nicotine was $\mathrm{m} / \mathrm{z} 84,162$.

\section{Results and Discussion}

\section{Selection of the filter of the sampler}

We have examined the kinds of the collection filter. Generally, glass fiber filter GB-100 was used for collection of aerosols. But collection amounts of duplicate samples were sometimes varied significantly. For example, nicotine amounts collected were 37 and $150 \mu \mathrm{g} / \mathrm{sampler}$ in the smoking room. Then we have investigated variations of the recoveries from alternative filters, thinner glass fiber filter GA-55 and quarts fiber filter QR-100. The recovery test was performed as follows: 5 filters were dipped with sodium hydrogen sulfate solution, and dried. After adding aliquot of nicotine standard solution in the filter, the nicotine in the sample was analyzed as mentioned below. The result was shown in Figure 1. This shows that recoveries from these filters tested were $49 \pm$ $27 \%$ for GA-55 and $94 \pm 11 \%$ for QR-100. Therefore, we selected QR100 for further investigation.

An advantage of this method was simple extraction procedure. We can treat many samples successively, and no organic solvent was necessary.

\section{Selection of the HPLC column}

To separate nicotine bisulfate by HPLC, we have examined the normal phase HILIC column, reverse phase ODS column, and a cation exchange column as the HPLC column [12-16]. When we used HILIC or ODS column, nicotine was eluted in 1 minute, and there is problems in the peak shape at large injection volume. Then we have tested a cation exchange column, Zorbax 300-SCX. Figure 2 shows the HPLC chromatograms of the sample extract, blank and nicotine standard solution. A retention time of nicotine was about 4 minutes, and the peak shape was excellent with the injection volume of $100 \mu \mathrm{L}$ which is 50 to 100 times large compared to that of 1 or $2 \mu \mathrm{L}$ used in GC. The sensitivity of a HPLC / DAD method of $0.2 \mathrm{ng}$ were worse than that of GC/MS of $0.04 \mathrm{ng}$, overall sensitivity of the HPLC method was 10 times more sensitive techniques than that of GC/MS.

\section{Calibration curve}

The nicotine calibration curves of the nicotine were also excellent, and the analysis of nicotine by this method shows a sufficient response in a wide range.

When we analyzed nicotine by GC/MS, the large deviation from the linear correlation between injection amount of nicotine and response was observed [17-19]. In this case, nicotine peak became small and broad when the injection amount decreased. This broadening of GC/ MS peak lead to larger uncertainties in the determination of nicotine. On the other hand, HPLC peak of nicotine sulfate didn't be broad, which lead to improve reliability of determination.

\section{Stability of nicotine bisulfate during sampling}

To avoid volatilization of nicotine during sampling, we collected nicotine as bisulfate. We have test the stability of nicotine bisulfate whether it was volatile or not during sampling. Figure 3 shows the result of stability of nicotine bisulfate. No significant difference of collection amount was observed after 24 hours. This suggests that nicotine sulfate were kept on the filter for more than $24 \mathrm{hrs}$. Saito and Seto reported that no nicotine was recovered from XAD tube when they drew air by 

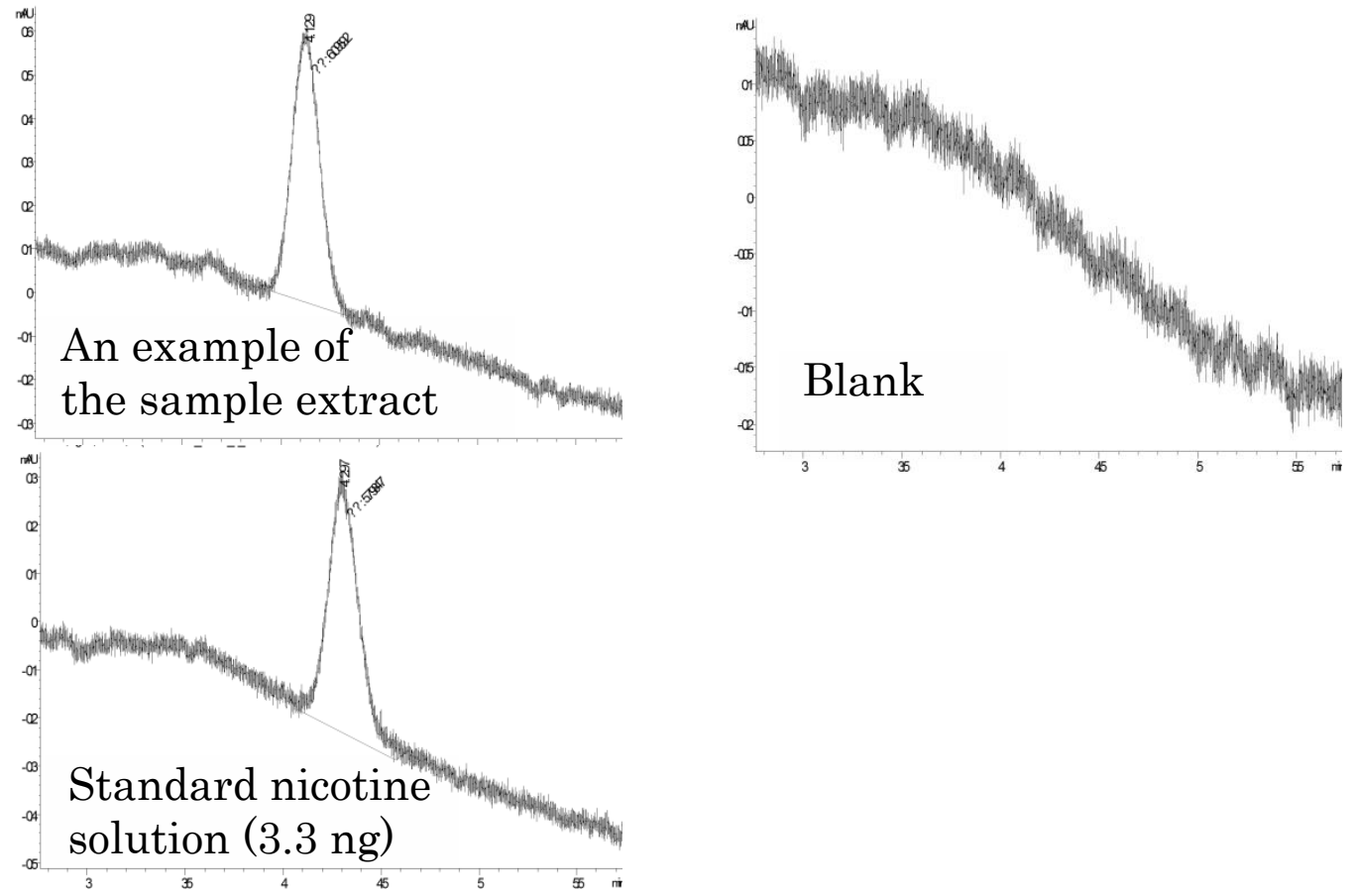

Figure 1: HPLC chromatograms of sample extract, blank and nicotine standard solution.

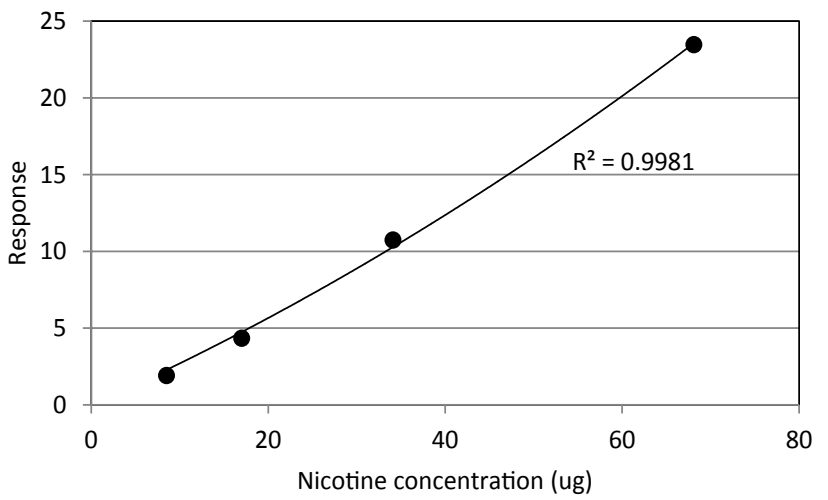

Figure 2: Calibration curve of nicotine determined by HPLC/spectrophotometry.

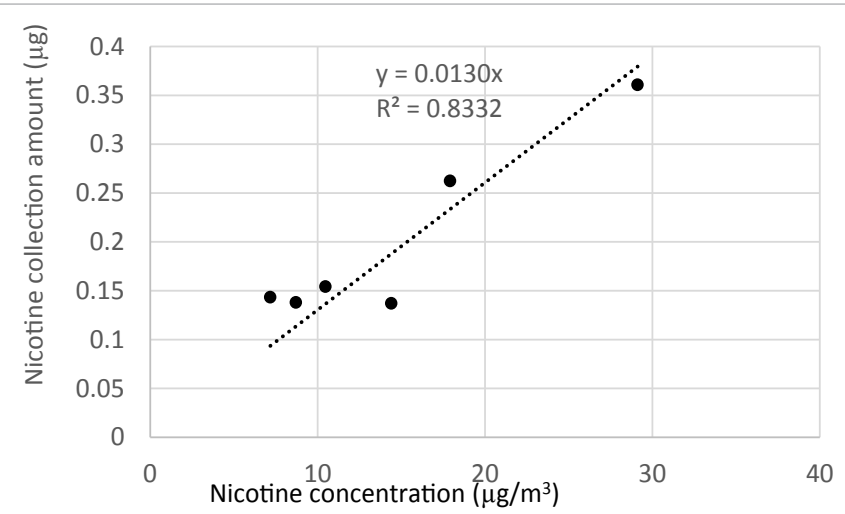

Figure 3: Correlation between nicotine concentrations in air collected by active sampler and nicotine collection amount by the passive sampler.

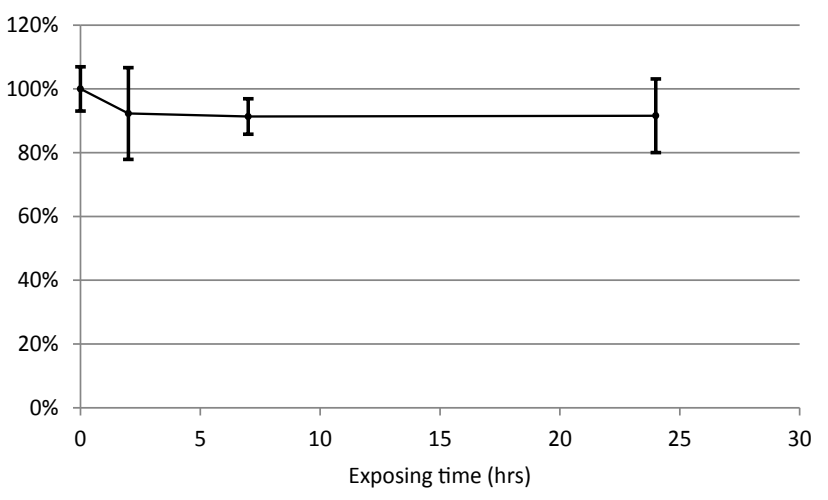

Figure 4: Stability of nicotine sulfate on the filter in the clean outdoor air

$50 \mathrm{~mL} \mathrm{~min}^{-1}$ for 24 hours [7]. In this case, nicotine may evaporate and passed through the tube or degrade by hydroxyl radicals [20].

It was also suggested that ambient ozone was not affect nicotine bisulfate. This means that nicotine sulfate was stable in the environment which was the advantages of this method.

\section{Correlation between collection amounts of nicotine by the passive sampler and its air concentration}

We have collected nicotine by the passive sampler and the active sampler simultaneously, and the results were compared, showing the correlation between collection amount by the passive sampler for an hour and air concentration of nicotine. The significant correlation $(\mathrm{r}$ $=0.9128$ ) was found between them. The deviations from regression line were found, this was due to broad peak of nicotine in GC/MS determination. 
Citation: Amagai T, Bai H, Wang Q, Miyake Y, Noguchi M , et al. (2015) Determination of Nicotine Exposure Using Passive Sampler and High Performance Liquid Chromatography. Pharm Anal Acta 6: 399. doi:10.4172/21532435.1000399

The sampling rate (SR) of nicotine by the passive sampler was calculated as [19]:

\section{$\mathrm{SR}=\mathrm{m} / \mathrm{ct}$}

where $\mathrm{m}(\mu \mathrm{g})$ is the nicotine amounts collected by the passive sampler;

$\mathrm{t}$ (min) was sampling time;

$\mathrm{c}\left(\mu \mathrm{g} \mathrm{m}^{-3}\right)$ was the average air concentration of nicotine.

The SR value was $9.03 \pm 0.63\left(\mathrm{~mL} \mathrm{~min}^{-1}\right)$, which was calculated from the slope of the regression line. This value was larger than that of tube type VOC sampler, smaller than that of badge type VOC sampler [20] (Figure 4).

This study suggests that nicotine determination methods developed including collection by sodium hydrogen sulfate impregnated filter, extraction with distilled water and separation analysis by HPLC / spectrophotometry can be applied for determining personal exposure of nicotine.

\section{Conclusion}

We have developed the determination method for nicotine exposure by using passive sampler and HPLC. It was reported that nicotine amounts decrease considerably during sampling when an adsorbent tube was used for collecting nicotine. The sodium bisulfate impregnated filter for nicotine collection has an advantage of preventing the decrease of nicotine. This collection method, however, needs neutralization and liquid-liquid extraction of nicotine when GC/MS was used for separation and analysis. The developed method included determination of nicotine sulfate directly by HPLC. This method could save time and a reagent. In addition, many samples can be treated simultaneously.

\section{Acknowledgement}

The authors are grateful to Dr. Yukio Yanagisawa, Principal of the Kaisei Academy for his suggestion. This study was supported in part by Smoking Research Foundation.

\section{Financial Supports}

This study was supported in part by Smoking Research Foundation. Dr. Amagai report grants from Smoking Research Foundation, during the conduct of the study; grants from Ministry of Health, Labour and Welfare, grants from Sibata Science and Technology, outside the submitted work (The ICMJE Conflict of Interest form).

\section{References}

1. Shinsugi C, Stickley A, Konishi S, Ng CF, Watanabe C (2015) Seasonality of child and adolescent injury mortality in Japan, 2000-2010. Environ Health Prev Med 20: 36-43.

2. (2004) IARC Monographs on the evaluation of carcinogenic risks to humans, International agency for research on cancer.

3. Phillips K, Bentley MC (2001) Seasonal assessment of environmental tobacco smoke and respirable suspended particle exposures for nonsmokers in Bremen using personal monitoring. Environ Int 27: 69-85.

4. Pandey SK, Kim KH (2010) A review of environmental tobacco smoke and its determination in air. Trends Anal Chem 29: 804-819.

5. Fu M, Martínez-Sánchez JM, Galán I, Pérez-Ríos M, Sureda X, et al. (2013) Variability in the correlation between nicotine and PM2.5 as airborne markers of second-hand smoke exposure. Environ Res 127: 49-55.
6. (2003) ISO 18145 Environmental tobacco smoke - Determination of vapor phase nicotine and 3-ethenylpyridine in air - gas chromatographic method International organization for standardization, ISO, Switzerland.

7. Saito I, Seto H (2007) Measurement of nicotine in indoor air collected by alkaline-coated solid phase cartridge followed by GC-MS analysis. J Health Sci 53: 53-59.

8. Brown RH (2000) Monitoring the ambient environment with diffusive samplers: Theory and practical considerations. J Environ Monit 2: 1-9.

9. Winberry WT Jr, Forehand L, Murphy NT, Ceroli A, Phinney B, et al. (1990) Determination of nicotine in indoor air using treated filter cassettes. Compendium of methods for the determination of air pollutants in indoor air. Method IP-2B United States Environmental Protection Agency, Research Triangle Park, NC.

10. Khanh Huynh C, Moix JB, Dubuis A (2008) [Development and application of the passive smoking monitor MoNIC]. Rev Med Suisse 4: 430-433.

11. 11. Rajkumar S, Huynh CK, Bauer GF, Hoffmann S, Röösli M (2013) Impact of a smoking ban in hospitality venues on second hand smoke exposure: a comparison of exposure assessment methods. BMC Public Health 13: 536.

12. Carlisle MR, Chicoine ML, Wygant MB (1992) A stability-indicating highperformance liquid chromatographic assay for nicotine in transdermal patches. Int J Pharmaceutics 80: 227-242.

13. Tambwekar KR, Kakariya RB, Garg S (2003) A validated high performance liquid chromatographic method for analysis of nicotine in pure form and from formulations. J Pharm Biomed Anal 32: 441-450.

14. Lauterbach JH, Bao M, Joza PJ, Rickert WS (2010) Free-base nicotine in tobacco products. Part I. Determination of free-base nicotine in the particulate phase of mainstream cigarette smoke and the relevance of these findings to product design parameters. Regul Toxicol Pharmacol 58: 45-63.

15. Ciolino LA, Turner JA, McCauley HA, Smallwood AW, Yi TY (1999) Optimization study for the reversed-phase ion-pair liquid chromatographic determination of nicotine in commercial tobacco products. J Chromatogr A 852: 451-463.

16. Piller M, Gilch G, Scherer G, Scherer M (2014) Simple, fast and sensitive LCMS/MS analysis for the simultaneous quantification of nicotine and 10 of its major metabolites. J Chromatogr B Analyt Technol Biomed Life Sci 951-952: major

17. Amagai T, Bai H, Miyake $\mathrm{Y}$ (2014) Analysis of nicotine exposure by the passive sampler/ HPLC method. The 11th Japan-China International Symposium on Health Sciences 5-6.

18. Watson CH, Trommel JS, Ashley DL (2004) Solid-phase micro extraction-based approach to determine free-base nicotine in trapped main-stream cigarette smoke total particulate matter. J Agric Food Chem 52: 7240-7245.

19. Crump D (1999) Application of diffusive samplers, In: Organic Indoor Air Pollutants, Wiley-VCH : 57-72.

20. Walgraeve C, Demeestere K, Dewulf J, Van Huffel K, Van Langenhove H (2011) Uptake rate behavior of tube-type passive samplers for volatile organic compounds under controlled atmospheric conditions. Atmos Environ 45: 5872 5879 . 